\title{
Equivalent Channel Parameters for Polar Coded Cooperative Relaying
}

\author{
Xuan Ma, Meng Zhu, Lixin Li, Xiaocong Wen, Jiaying Yin, Jing Li \\ School of Electronics and Information, \\ Northwestern Polytechnical University, Xi'an, Shaanxi 710129, China \\ E-mail:maxuan@mail.nwpu.edu.cn
}

\begin{abstract}
In a polar coded cooperative relay system, the channel parameters play a very important role in the overall system performance. In order to find the optimal channel parameter which is suitable for polar encoding and decoding, and to optimize the system performance, a equivalent channel parameter (ECP) value which is universal to the $\mathrm{AF}$ cooperative relay system is given by rigorous mathematical derivation in this paper. The paper also analyzes the influence of the channel parameters on the ECP in a special case, and gives the simulation results.
\end{abstract}

Keywords: Polar codes; cooperative relaying; equivalent channel parameter (ECP).

\section{Introduction}

Cooperative relaying using relay channels [1] is a technology that has been widely used in wireless communication networks, which can significantly improve system capacity and error rate performance. The cooperative relaying obtains the diversity gain through the cooperation of each node, and then achieves the effect of a kind of virtual MIMO, which can be regarded as a special channel polarization process. Polar codes based on channel polarization, introduced by Arikan in [2], is the first coding scheme that theoretically achieve symmetric capacity of binary-input discrete memoryless channels (BDMCs). The excellent performance of the polar codes makes it attractive for scholars all over the word. At present, polar codes have been applied in many fields, e.g. image transmission [3], wiretap channel [4] and quantum channel [5], which have proved to be very advantageous.

Based on the similar channel polarization characteristics, the combination of the polar codes and the cooperative relay channel will result in better error performance and channel capacity. It is known that the polar codes is combined with the relay channel for the first time in the paper [6]. The paper describes the implementation of symmetric binary-input capacity in the physical degraded relay channel. After that, there are a lot of research on the combination of polar codes and relay channel, e.g. [7][8][9].

It is known that the polar codes are constructed based on the channel polarization characteristics. Therefore, channel parameters such as signal-to-noise ratio (SNR), noise variance, and so on, have a great impact on their performance. In cooperative relay systems with similar channel polarization, the influence of channel parameters is more obvious. In the usual research, scholars have adopted a simpler way, the default relay system parameters of the same channel. In practice, this setting is unrealistic, and has greater damage to the polar performance. In this paper, the ECP which can optimize the performance of the system are found from the general amplify and forward (AF) model using Rayleigh channel, and the ECP affected analysis in Gaussian channels are given.

The paper is organized as follows: In section 2, we briefly describe the relay system model based on polar codes in Rayleigh channel. The derivation of ECP is given in section 3. At the same time, the influence of ECP on Gaussian channel and its simulation analysis are also given in Section 3. Some concluding remarks are provided in section 4 .

\section{System model}

Figure 1 shows the system chart of the polar coding scheme based on the AF protocol. In the first stage, at the source node S, the information is first polar encoded and then modulated and broadcasted; in the second stage, the relay $\mathrm{R}$ amplifies the received signal with amplification factor $\alpha$, and then forwards it to the destination node $\mathrm{D}$; $\mathrm{D}$ will be combined to decode the output of the two signals. 


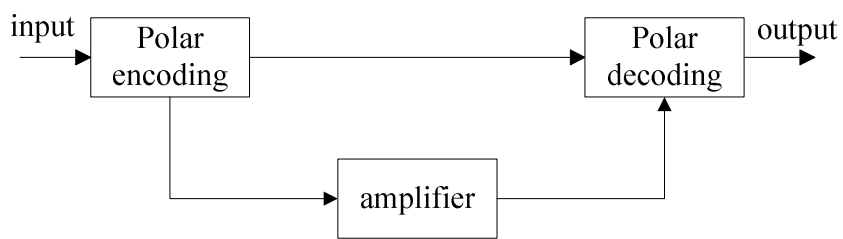

Figure 1. Polar coding scheme based on the AF protocol

Figure 2 shows the corresponding mathematical model of the cooperative polar coding system under AF protocol. In this model, all nodes are single-antenna systems. Assume that the signal transmission power at $\mathrm{S}$ and $\mathrm{R}$ is $P_{S}$ and $P_{R}$, respectively. Each channel corresponds to the channel fading coefficient and noise is $h_{i j}$ and $n_{i j}$ $(i, j=S, R, D)$ respectively.

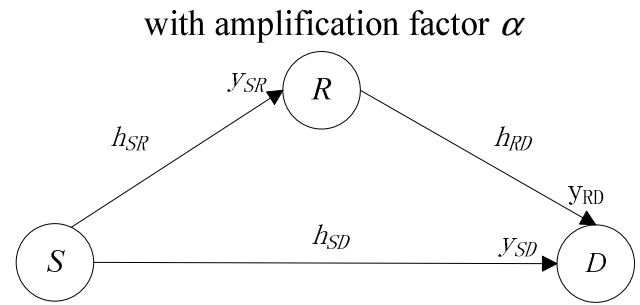

Figure 2. Mathematical model of single relay polar code cooperative system

As shown in Figure 2, $x$ denotes a transmission signal, and $y_{i j}(i, j=S, R, D)$ denotes a reception signal the received signal can be expressed as

$$
\begin{aligned}
y_{S R} & =h_{S R} x+n_{S R} \\
y_{R D} & =h_{R D} \cdot \alpha y_{R}+n_{R D} \\
& =\alpha h_{S R} h_{R D} x+\alpha h_{R D} n_{S R}+n_{R D} \\
y_{S D} & =h_{S D} x+n_{S D}
\end{aligned}
$$

The detailed derivation of the combined signal is given in the next section.

\section{The Derivation of ECP}

In this part, we derive the system ECP from the known information of the system in formula level, which can make the system get the best BER performance. The ECP specific derivation process is described in detail below.

\subsection{Derivation of the component parameters}

\section{a) Channel SR}

Using $\sigma_{1}^{2}$ denotes the noise variance on the channel SR, and $S N R_{1}$ represents the SNR at the signal receiving side, combing (1), there is

$$
S N R_{1}=\frac{\left|h_{S R}\right|^{2} P_{s}}{\sigma_{1}^{2}} \Rightarrow \sigma_{1}=\sqrt{\frac{\left|h_{S R}\right|^{2} P_{s}}{S N R_{1}}} .
$$

b) Amplification times of relay node.

The transmit signal of relay node is $\alpha y_{R}$, so the signal power

$$
P_{R}=\alpha^{2}\left|h_{S R}\right|^{2} P_{s}+\alpha^{2} \sigma_{1}^{2} \text {. }
$$

Therefore,

c) Channel RD.

$$
\alpha=\sqrt{\frac{P_{R}}{\left|h_{S R}\right|^{2} P_{s}+\sigma_{1}^{2}}}
$$

Similarly, Using $\sigma_{2}^{2}$ denotes the noise variance on the channel $\mathrm{RD}$, and $S N R_{2}$ represents the SNR at the signal receiving side, combing (2)(6), there is 


$$
\begin{gathered}
S N R_{2}=\frac{\left|\alpha h_{S R} h_{R D}\right|^{2} P_{s}}{\left|h_{R D}\right|^{2} \cdot \alpha^{2} \sigma_{1}^{2}+\sigma_{2}^{2}} \\
\Rightarrow \sigma_{2}=\sqrt{\frac{\alpha^{2}\left|h_{R D}\right|^{2}\left|h_{S R}\right|^{2} P_{s}}{S N R_{2}}-\left|h_{R D}\right|^{2} \cdot \alpha^{2} \sigma_{1}^{2}}
\end{gathered}
$$

d) Channel SD.

In the channel SD, the noise variance and SNR are denoted by $\sigma_{3}^{2}$ and $S N R_{3}$, respectively. The same can be concluded as follows

$$
\begin{gathered}
S N R_{3}=\frac{\left|h_{S D}\right|^{2} P_{s}}{\sigma_{3}^{2}} \\
\Rightarrow \sigma_{3}=\sqrt{\frac{\left|h_{S D}\right|^{2} P_{s}}{S N R_{3}}}
\end{gathered}
$$

So far, we obtain the channel parameters needed to derive the ECP for all three channels. In the next step, we will use these parameters to get what we want ECP.

\subsection{ECP derivation}

At the destination node $\mathrm{D}$, we have received the relay channel and the direct channel transmission information $y_{R D}$ and $y_{S D}$, in practice, we need to merge according to certain rules to combine the two signals, and then perform the decoding operation. In the process of decoding the combined signal, the noise variance plays a very important role in the decoding performance. Therefore, we derive the EPC for the whole system based on the component parameters obtained in 3.1, in order to obtain the optimal performance of the system. Specific derivation process is as follows.

First of all, we combine the received signals using maximum-ratio-combing (MRC). As known

so there is

$$
\hat{y}=\frac{h_{0}^{*} y_{0}+h_{1}^{*} y_{1}+\cdots+h_{n}^{*} y_{n}}{\left|h_{0}\right|^{2}+\left|h_{1}\right|^{2}+\cdots+\left|h_{n}\right|^{2}},
$$

$$
y_{M R C}=\frac{h_{S D}^{*} y_{S D}+\alpha h_{S R}^{*} h_{R D}^{*} y_{R D}}{\left|h_{S D}\right|^{2}+\left|\alpha h_{S R} h_{R D}\right|^{2}} .
$$

Combining equations (2) (3), (12) can be rewritten as

$$
\begin{aligned}
y_{M R C}= & \frac{h_{S D}^{*} h_{S D} x+h_{S D}^{*} n_{S D}+\left(\alpha h_{S R} h_{R D}\right)^{*}\left[\alpha h_{S R} h_{R D} x+\alpha h_{R D} n_{S R}+n_{R D}\right]}{\left|h_{S D}\right|^{2}+\left|\alpha h_{S R} h_{R D}\right|^{2}} \\
& =\frac{\left|h_{S D}\right|^{2} x+h_{S D}^{*} n_{S D}+\alpha^{2}\left|h_{S R}\right|^{2}\left|h_{R D}\right|^{2} x+\alpha^{2}\left|h_{R D}\right|^{2} h_{S R}^{*} n_{S R}+\alpha h_{S R}^{*} h_{R D}^{*} n_{R D}}{\left|h_{S D}\right|^{2}+\alpha^{2}\left|h_{S R}\right|^{2}\left|h_{R D}\right|^{2}} \\
& =\frac{\left(\left|h_{S D}\right|^{2}+\alpha^{2}\left|h_{S R}\right|^{2}\left|h_{R D}\right|^{2}\right) x+h_{S D}^{*} n_{S D}+\alpha h_{S R}^{*} h_{R D}^{*} n_{R D}+\alpha^{2}\left|h_{R D}\right|^{2} h_{S R}^{*} n_{S R}}{\left|h_{S D}\right|^{2}+\alpha^{2}\left|h_{S R}\right|^{2}\left|h_{R D}\right|^{2}} \\
= & \mathrm{x}+\frac{h_{S D}^{*} n_{S D}+\alpha h_{S R}^{*} h_{R D}^{*} n_{R D}+\alpha^{2}\left|h_{R D}\right|^{2} h_{S R}^{*} n_{S R}}{\left|h_{S D}\right|^{2}+\alpha^{2}\left|h_{S R}\right|^{2}\left|h_{R D}\right|^{2}}
\end{aligned}
$$

Therefore, the equivalent SNR parameter of the total signal can be obtained

$$
S N R_{e q}=\frac{P_{s}}{\frac{\left|h_{S D}\right|^{2} \sigma_{3}^{2}+\alpha^{2}\left|h_{S R}\right|^{2}\left|h_{R D}\right|^{2} \sigma_{2}^{2}+\alpha^{4}\left|h_{R D}\right|^{4}\left|h_{S R}\right|^{2} \sigma_{1}^{2}}{\left(\left|h_{S D}\right|^{2}+\alpha^{2}\left|h_{S R}\right|^{2}\left|h_{R D}\right|^{2}\right)^{2}}}
$$

and the equivalent noise variance parameter is

$$
\sigma_{e q}^{2}=\frac{\left|h_{S D}\right|^{2} \sigma_{3}^{2}+\alpha^{2}\left|h_{S R}\right|^{2}\left|h_{R D}\right|^{2}\left(\sigma_{2}^{2}+\alpha^{2}\left|h_{R D}\right|^{2} \sigma_{1}^{2}\right)}{\left(\left|h_{S D}\right|^{2}+\alpha^{2}\left|h_{S R}\right|^{2}\left|h_{R D}\right|^{2}\right)^{2}} .
$$

That is, $\sigma_{e q}$ is composed of $\sigma_{1}, \sigma_{2}, \sigma_{3}$ according to the above function relationship. Furthermore, substituting $\sigma_{1}, \sigma_{2}, \sigma_{3}$ obtained by the formula (4) (8) (10) into the formula (18), respectively, there is 


$$
\begin{aligned}
\sigma_{e q}^{2} & =\frac{\frac{\left|h_{S D}\right|^{2} \cdot\left|h_{S D}\right|^{2} P_{s}}{S N R_{3}} \sigma_{3}^{2}+\alpha^{2}\left|h_{S R}\right|^{2}\left|h_{R D}\right|^{2}\left(\frac{\alpha^{2}\left|h_{R D}\right|^{2}\left|h_{S R}\right|^{2} P_{s}}{S N R_{1}}+\frac{\alpha^{2}\left|h_{R D}\right|^{2}\left|h_{S R}\right|^{2} P_{s}}{S N R_{2}}-\frac{\alpha^{2}\left|h_{R D}\right|^{2}\left|h_{S R}\right|^{2} P_{s}}{S N R_{1}}\right)}{\left(\left|h_{S D}\right|^{2}+\alpha^{2}\left|h_{S R}\right|^{2}\left|h_{R D}\right|^{2}\right)^{2}} \\
& =\frac{\frac{\left|h_{S D}\right|^{4} P_{s}}{S N R_{3}}+\alpha^{2}\left|h_{S R}\right|^{2}\left|h_{R D}\right|^{2} \cdot \frac{\alpha^{2}\left|h_{R D}\right|^{2}\left|h_{S R}\right|^{2} P_{s}}{S N R_{2}}}{\left(\left|h_{S D}\right|^{2}+\alpha^{2}\left|h_{S R}\right|^{2}\left|h_{R D}\right|^{2}\right)^{2}} \\
& =\frac{\frac{\left(\left.h_{S D}\right|^{2}\right)^{2}}{S N R_{3}}+\frac{\left(\alpha^{2}\left|h_{R D}\right|^{2}\left|h_{S R}\right|^{2}\right)^{2}}{S N R_{2}}}{\left(\left|h_{S D}\right|^{2}+\alpha^{2}\left|h_{S R}\right|^{2}\left|h_{R D}\right|^{2}\right)^{2}} \cdot P_{s} .
\end{aligned}
$$

Further, from (4) (6),

$$
\alpha^{2}=\frac{P_{R}}{\left|h_{S R}\right|^{2} P_{s}+\sigma_{1}^{2}}=\frac{P_{R}}{\left|h_{S R}\right|^{2} P_{s}\left(1+\frac{1}{S N R_{1}}\right)}
$$

So we have

$$
\alpha^{2}\left|h_{R D}\right|^{2}\left|h_{S R}\right|^{2}=\frac{P_{R}}{P_{s} \cdot\left|h_{S R}\right|^{2}\left(1+\frac{1}{S N R_{1}}\right)} \cdot\left|h_{R D}\right|^{2}\left|h_{S R}\right|^{2}=\frac{\left|h_{R D}\right|^{2} P_{R}}{P_{s}} \cdot \frac{S N R_{1}}{1+S N R_{1}}
$$

Combinations (23), (21) can be written as

And there is

$$
\sigma_{e q}^{2}=\frac{\frac{\left|h_{S D}\right|^{4}}{S N R_{3}}+\left[\frac{\left|h_{R D}\right|^{4} P_{R}^{2}}{P_{s}^{2}} \cdot\left(\frac{S N R_{1}}{1+S N R_{1}}\right)^{2}\right] / S N R_{2}}{\left(\left|h_{S D}\right|^{2}+\frac{\left|h_{R D}\right|^{2} P_{R}}{P_{s}} \cdot \frac{S N R_{1}}{1+S N R_{1}}\right)^{2}} \cdot P_{s}
$$

$$
S N R_{e q}=\frac{P_{s}}{\sigma_{e q}^{2}}=\frac{\left(\left|h_{S D}\right|^{2}+\frac{\left|h_{R D}\right|^{2} P_{R}}{P_{s}} \cdot \frac{S N R_{1}}{1+S N R_{1}}\right)^{2}}{\frac{\left|h_{S D}\right|^{4}}{S N R_{3}}+\left[\frac{\left|h_{R D}\right|^{4} P_{R}^{2}}{P_{s}^{2}} \cdot\left(\frac{S N R_{1}}{1+S N R_{1}}\right)^{2}\right] / S N R_{2}}
$$

From (25), it can be seen that $S N R_{e q}$ is composed of $S N R_{1}, S N R_{2}$ and $S N R_{3}$ according to the defined function relationship. In the next section, we will analyze the channel fading coefficient of 1 (i.e. with Gaussian channel), the impact of each component channel parameters on the ECP.

\subsection{ECP affected analysis in Gaussian channels}

In the previous section, we present the ECP formulation for any Rayleigh fading channel. Using the ECP, the system can achieve better performance. In this section, we simplify the system model by defining all the channel fading coefficients as $1, P_{S}=P_{R}$ and the amplification factor is also 1 , in order to more intuitively express the influence of each component parameter on ECP. In this case, the influence of the component channel parameters on ECP is expressed as follows.

In this particular case, there is

$$
\begin{aligned}
S N R_{e q}=\frac{P_{s}}{\sigma^{2}}= & \frac{\left(1+\frac{S N R_{1}}{1+S N R_{1}}\right)^{2}}{\frac{1}{S N R_{3}}+\left(\frac{S N R_{1}}{1+S N R_{1}}\right)^{2} / S N R_{2}} \\
\sigma_{e q}^{2} & =\frac{\sigma_{3}^{2}+\sigma_{2}^{2}+\sigma_{1}^{2}}{4}
\end{aligned}
$$

For $S N R_{e q}$ and $\sigma_{e q}$ have a one-to-one relationship $S N R_{e q}=P_{S} / \sigma_{e q}^{2}$, so we will take $S N R_{e q}$ as an example. Figure 3, Figure 4, Figure 5 show the influence of the other two component channel parameters on $S N R_{e q}$ when fixed $S N R_{1}, S N R_{2}$ and $S N R_{3}$ respectively. 


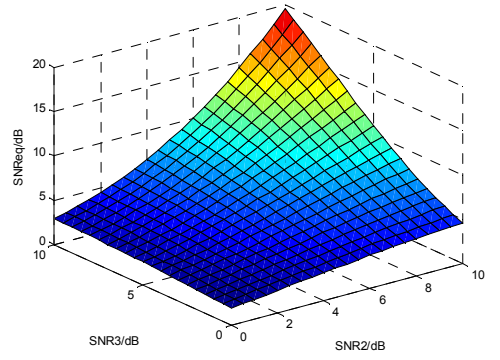

(a) $S N R_{1}=5 d B$

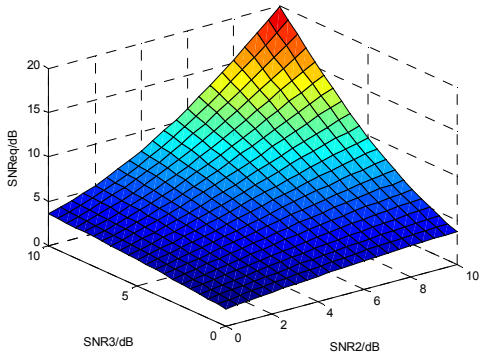

(b) $S N R_{1}=15 \mathrm{~dB}$

Figure 3. The effect of $S N R_{1}, S N R_{2}$ and $S N R_{3}$ on $S N R_{e q}$ when fixed $S N R_{1}$

Figure 3 shows the influence of the other two parameters on the $S N R_{e q}$ when $S N R_{1}$ is taken. The purpose of selecting the two values $(5 \mathrm{~dB}$ and $10 \mathrm{~dB})$ is to study the influence of the other two parameters on the ECP when the fixed channel parameters are within and outside the fluctuation range. We selected the same parameters in the next two simulations. From Figure 3, we can see that when fixed $S N R_{1}, S N R_{e q}$ increases with the increase of $S N R_{2}$ and $S N R_{3}$, and the effect of $S N R_{2}$ and $S N R_{3}$ on $S N R_{e q}$ is approximately equal. In addition, we were surprised to find that in this case, the change in the value of $S N R_{1}$ has almost no effect on the ECP.

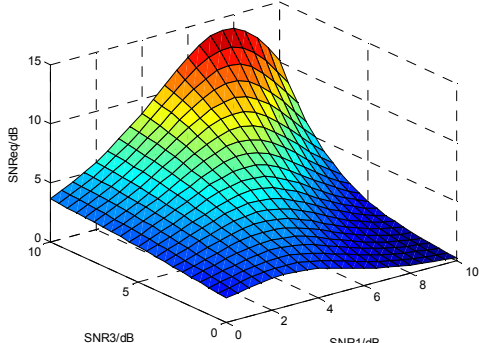

(a) $S N R_{2}=5 d B$

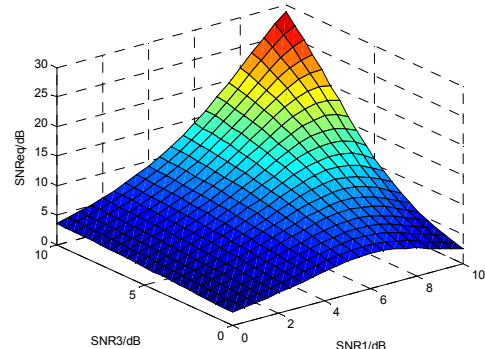

(b) $S N R_{2}=15 d B$

Figure 4. The effect of $S N R_{1}, S N R_{2}$ and $S N R_{3}$ on $S N R_{e q}$ when fixed $S N R_{2}$

Figure 4 shows a very interesting phenomenon. That is, when $S N R_{2}$ is within the range of $S N R_{1}$ and $S N R_{3}$, $S N R_{e q}$ increases with the increase of $S N R_{3}$, but not with the increase of $S N R_{1}$ presents an increasing trend. There will be a maximum value, and when the value of $S N R_{1}$ and $S N R_{2}$ are approximately equal to achieve this extreme. What's more, when the value of $S N R_{2}$ exceeds the range of $S N R_{1}$ and $S N R_{3}$, this phenomenon does not exist.

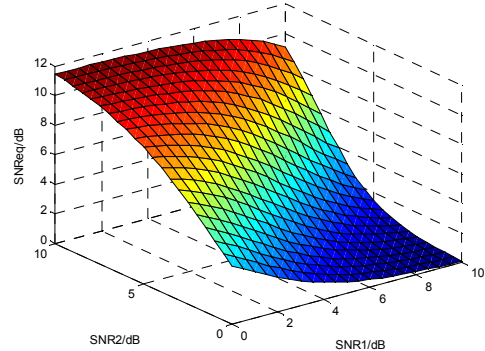

(a) $S N R_{3}=5 d B$

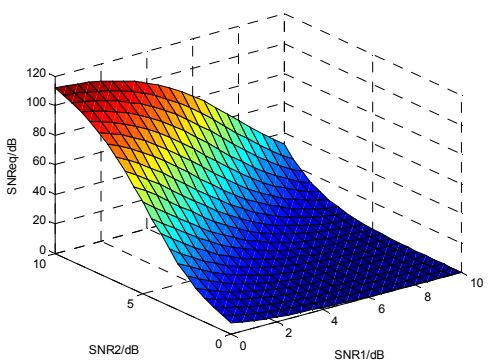

(b) $S N R_{3}=15 d B$

Figure 5. The effect of $S N R_{1}, S N R_{2}$ and $S N R_{3}$ on $S N R_{e q}$ when fixed $S N R_{3}$

The effect of $S N R_{1}, S N R_{2}$ and $S N R_{3}$ on $S N R_{e q}$ when fixed $S N R_{3}$ is shown in Figure 5. As shown in the figure, when fixed $S N R_{3}$, the appropriate reduction of $S N R_{1}$ will get a larger $S N R_{e q}$. And for different values of $S N R_{3}$, the impact of ECP is very significant. 


\section{Conclusion}

The performance of polar codes based on channel polarization is very sensitive to channel parameters, especially in cooperative relay channels. And in general, it is not practical to set the relay channel and direct passing channel parameter to be the same. In order to find the optimal channel parameter which is suitable for polar encoding and decoding, and to optimize the system performance, a ECP value which is universal to the AF cooperative relay system is given by rigorous mathematical derivation. This ECP varies with the variation of the channel parameters (arbitrary) of the system. The paper analyzes the influence of the channel parameters on the ECP in a special case, and gives the simulation results.

\section{Acknowledgments}

This work is supported by Shaanxi Province natural science basic research plan surface project (No. 2016JM6062), by China Aerospace Science and Technology Corporation Aerospace Science and Technology Innovation Fund funded project, by the Seed Foundation of Innovation and Creation for Graduate Students in Northwestern Polytechnical University.

\section{References}

[1] E. C. van der Meulen. Three-terminal communication channels. Advance in Applied Probability, 1971: 120-154.

[2] E. Arikan. Channel Polarization: A method for Constructing Capacity-achieving Codes for Symmetric Binary-input Memoryless Channels. IEEE Transactions on Information Theory, 2009: 3051-3073.

[3] Peng Shi, Zhifang Zheng, Longyan Gong, Shengmei Zhao. Performance of Polar Code in Image Transmission. Journal of Nanjing University of Posts and Telecommunications (Natural Science), 2014: 65-71.

[4] Fantao Wu, Chao Xing, Shengmei Zhao, Feng Gao. Encrypted Polar Codes for Wiretap Channel. 2012 2nd International Conference on Computer Science and Network Technology (ICCSNT). IEEE. 2012:579-583.

[5] Mark M. Wilde, Saikat Guha. Polar codes for classical-quantum channels. IEEE Transactions on Information Theory, 2013: 1175-1187.

[6] M. Andersson, V. Rathi, R. Thobaben, J. Kliewer and M. Skoglund. Nested Polar Codes for Wiretap and Relay Channels. IEEE Communications Letters, 2010:752-754.

[7] R Blasco-Serrano, R Thobaben, M Andersson and V Rathi. Polar Codes for Cooperative Relaying. Communications IEEE Transactions on, 2012:3263-3273.

[8] DS Karas, KN Pappi, GK Karagiannidis. Smart Decode-and-Forward Relaying witn Polar Codes. Wireless Communications Letters IEEE, 2014:62-65.

[9] Lele Wang. Polar Coding for Relay Channels. IEEE International Symposium on Information Theory. IEEE. 2015:1532-1536. 